Revista Colombiana de Obstetricia y Ginecología Vol. 55 No.4 • $2004 \cdot(300-307)$

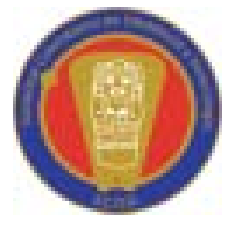

ARtículo De Revisión

\title{
MUERTE FETAL INEXPLICADA
}

\section{UINEXPLAINED FETAL DEATH}

Janer Sepúlveda, M.D.*, Eliana Maribel Quintero, M.D.**

Recibido: septiembre 15/2004 - Revisado: octubre 14/2004 - Aceptado: noviembre 22/2004

\section{RESUMEN}

El porcentaje de muertes fetales inexplicadas oscila entre un 21\% a 50\%; se define como la muerte que ocurre en fetos con edad gestacional mayor de 20 semanas o peso superior a $500 \mathrm{~g}$, en la cual ni la autopsia ni el examen histológico del cordón umbilical, placenta y membranas, se logra identificar la causa.

Los factores asociados con muerte fetal inexplicada son edad materna mayor de 35 años, sobrepeso, nivel educativo menor de 10 años, cigarrillo y bajo nivel socioeconómico, entre otros.

La muerte fetal se relaciona con enfermedades maternas, trombofilia, accidentes del cordón, alteraciones citogenéticas, metabólicas e infecciones congénitas, principalmente.

Se realizó una revisión de muerte fetal inexplicada.

Palabras clave: muerte fetal inexplicada, factores de riesgo, enfermedades maternas, trombofilias, cordón umbilical, infecciones congénitas.

\section{SUIMMARY}

Reports in the literature demonstrate that the percentage of unexplained fetal death occurs from 21 to $50 \%$. Unexplained fetal death is defined as the demise of the fetus occurring at gestational age beyond 20 weeks or fetal weight of more than

\footnotetext{
* Médico Especialista en Ginecología y Obstetricia. Profesor de Cátedra Titular. Universidad Industrial de Santander. Bucaramanga, Colombia. Correo electrónico: janersepulveda@yahoo.es

* Médica Especialista en Ginecología y Obstetricia. Profesora Asociada. Universidad Autónoma de Bucaramanga.
}

$500 \mathrm{~g}$, with a thorough autopsy of the fetus and histology examination of the umbilical cord, placenta, and membranes, fails to demonstrate the cause of death.

The following factors are associated with unexplained fetal death, maternal age beyond 35 years, overweight, ten years or less in a school, smoking, low socioeconomic status class and others.

Fetal deaths are related to maternal medical disease, thrombophilia, umbilical cord accidents, cytogenetics, metabolic and congenital infections.

The following is a review of the main aspects of unexplained fetal death.

Key words: unexplained fetal death, stillbirth, fetal loss, risk factors, maternal disease, thrombophilia, umbilical cord, congenital infections.

\section{INTRODUCCIÓN}

Con base en el elevado nivel de angustia y frustración que genera la pérdida de una gestación no sólo a los padres, sino también a los médicos tratantes involucrados en el manejo del embarazo, consideramos pertinente realizar una revisión de la literatura publicada al respecto a través de medios electrónicos, no sólo con la intención de proveer algunas ideas acerca del enfoque de manejo y estudio del evento en el mismo momento en que se presenta, sino también como un mecanismo de asesoría para los gineco-obstetras encargados de brindar una atención preconcepcional a la pareja que sufre este antecedente y desea una nueva gestación. 
Con base en las consideraciones previas, realizamos una búsqueda sistemática de la literatura publicada en idioma inglés a través de MEDLINE a partir de 1985, usando como palabras clave: fetal death, thrombophilia, risk factors, stillbirth, fetal loss.

De la búsqueda realizada se seleccionaron 51 artículos que a criterio de los autores cumplían con los objetivos de la revisión planteada por su contenido y enfoque. Una vez limitada la bibliografía se procedió a analizarla y depurarla para extraer conceptos que pudieran ser expuestos de manera crítica. Es de anotar que por la gran variedad de propuestas como factores de riesgo y como posibles mecanismos etiológicos de muerte fetal no fue posible generar un único lineamiento clasificatorio para la selección de la literatura disponible, la cual de cualquier manera no es muy extensa. De esta búsqueda se obtuvo la información que a continuación se presenta. Se describen los factores de riesgos asociados, las posibles causas que pueden producir una muerte fetal inexplicada (MFI), con especial énfasis en las trombofilias que cada día adquieren mayor importancia en la obstetricia y finalmente se emiten algunas recomendaciones acerca del tema.

Para situarnos en la problemática planteada, iniciamos limitando el concepto de MFI que se define como la que ocurre en edades gestacionales mayores de 20 semanas o en fetos con más de 500 $g$, que se presenta previo al inicio del trabajo de parto y en la que a pesar de una valoración anatomopatológica del cordón umbilical, las membranas, la placenta y el feto no se logra demostrar la causa de muerte. ${ }^{1,2}$

Aún hoy en día, a pesar de contar con modernas técnicas de análisis de laboratorio, la persistencia de casos de MFI sigue siendo elevada. Los reportes bibliográficos revisados muestran una incidencia que oscila entre el 21 y el $50 \%$ del total de casos de muerte fetal. ${ }^{1,2}$

\section{FACTORES DE RIESGO}

Una evaluación de la bibliografía publicada al respecto nos permite concluir que existen factores claramente identificados como asociados con una mayor incidencia de MFI como son:

\section{Edad materna}

Las mujeres mayores de 35 años tiene un mayor riesgo de desarrollar $\mathrm{MFI}^{1,3-9}$ tal como se muestra en la tabla 1, que presenta el OR obtenido por Froen y cols. ${ }^{2}$ Los embarazos en adolescentes están asociados con incremento de muerte neonatal temprana, mas no con MFI. ${ }^{10}$

\section{Paridad}

La paridad muestra reportes controvertidos al ser evaluada como factor de riesgo para MFI. Por un lado, reportes como el publicado por Huang y cols., ${ }^{11}$ que es un estudio de cohortes, concluyen que la nuliparidad y la multiparidad con más de tres gestaciones previas generan unos OR estadísticamente significativos como factores de riesgo para $\mathrm{MFI}^{11}$ (tabla 2).

En contraposición, Froen y cols., en su publicación no clasifican esta variable como factor de riesgo, tomando en cuenta que sus evaluaciones no le permiten emitir esto como una conclusión estadísticamente significativa. ${ }^{1}$

\section{Obesidad}

Índices de masa corporal mayor de 25 (sobrepeso en adelante) se correlacionan con 2,3 veces más el riesgo de $\mathrm{MFI}^{1,8,12-14}$ (tabla 3). Stephansson y cols.

\section{Tabla 1. Edad materna y el riesgo de MFI}

\begin{tabular}{ccc} 
Edad materna & OR ajustado & IC95\% \\
\hline$<25$ & 1,0 \\
\hline $25-30$ & 2,88 & $0,99-8,32$ \\
\hline $30-35$ & 2,85 & $0,95-8,62$ \\
\hline$>35$ & 5,09 & $1,32-19,57$ \\
\hline \multicolumn{3}{c}{ Tabla 2. Paridad y riesgo de MFI } \\
\hline Paridad & OR ajustado & IC95\% \\
\hline 0 & 1,74 \\
\hline $1-2$ & 1,0 grupo referencial \\
\hline $3-4$ & 2,01 & $1,26-2,4$ \\
\hline
\end{tabular}


cruzaron la ganancia de peso durante el embarazo con el riesgo de muerte fetal y no encontraron asociaciones significativas. ${ }^{14}$

\section{Hábito de fumar}

Froen y cols. (tabla 4) así como Sims y cols. publican en sus revisiones clínicas que madres fumadoras de más de diez cigarrillos por día presentan tres veces más riesgo de MFI cuando se comparan con no fumadoras. ${ }^{1,8}$

\section{Nivel educativo}

Los años de estudio cursados y su relación con la posibilidad de MFI fueron evaluados por Froen y cols. (tabla 5); concluyeron que las madres con niveles educativos equivalentes a doce años de estudio no presentan un factor de riesgo adicional para muerte fetal inexplicada, mientras que en aquellas con niveles educativos de menos de diez años, se evidencia un riesgo 3,7 veces mayor de presentarla; ${ }^{1}$ esta misma conclusión fue sustentada por Fifer y col. ${ }^{15}$

\section{Edad gestacional}

Yudkin y cols. en su estudio no sólo encuentran que a mayor edad gestacional existe un riesgo su-

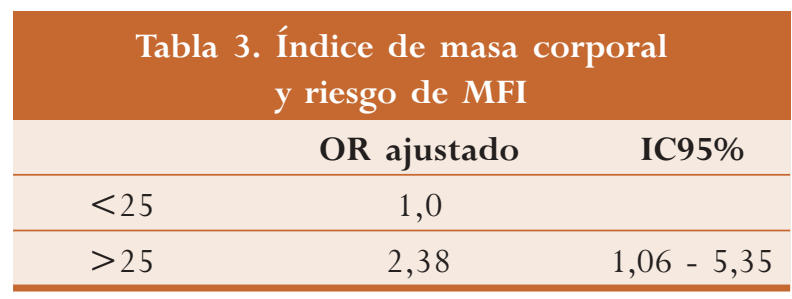

Tabla 4. Hábito de fumar y riesgo de MFI

\begin{tabular}{lcc} 
& OR ajustado & IC95\% \\
\hline No fumadora & 1,0 & \\
\hline$<10$ cigarrillos/día & 0,66 & $0,23-1,97$ \\
\hline$>10$ cigarrillos/día & 3,11 & $1,2-8,07$ \\
\hline
\end{tabular}

Tabla 5. Nivel educativo y riesgo de MFI

\begin{tabular}{lcc} 
Educación & OR ajustado & IC95\% \\
\hline$<10$ años & 3,77 & $1,50-9,48$ \\
\hline $10-12$ años & 1,63 & $0,73-3,65$ \\
\hline$>12$ años & 1,0 & \\
\hline
\end{tabular}

perior de MFI, sino de muerte fetal en general; concluyen que para las estadísticas revisadas por su grupo se observa un caso de MFI por cada 926 embarazos de 40 semanas. ${ }^{16}$ King y cols. también reportan esta asociación. ${ }^{17}$

\section{Otros}

Uno de los factores de riesgo más controvertidos en la MFI es haber presentado en una gestación previa este mismo diagnóstico. Cnattingius y cols. reportan un aumento de seis a diez veces el riesgo de repetir un segundo episodio de MFI en comparación con pacientes que no tienen este antecedente. ${ }^{18}$ Sin embargo, Froen y cols. no concuerdan con esta conclusión y descartan en su publicación la MFI previa como factor de riesgo importante. ${ }^{1}$

Como factores asociados se señalan las concentraciones de hemoglobina menores de $11,5 \mathrm{~g} / \mathrm{dl} \mathrm{y}$ mayores de 14,6 g/dl, ${ }^{19}$ así como pacientes que cursen con embarazos múltiples. ${ }^{20}$ El consumo excesivo de café, definido como ocho tazas o más por día, aumenta tres veces el riesgo de muerte fetal comparado con mujeres que no lo consumen (OR 3,0 con intervalo de confianza 1,5 - 5,9); ${ }^{21}$ también están asociados la ingestión de alcohol y el abuso de cocaína. ${ }^{8}$ El antecedente de cesárea en el primer embarazo también puede incrementar el riesgo de MFI, al parecer básicamente relacionado con ruptura uterina (OR 2,74 con intervalo de confianza de $1,74-4,3) .^{22}$

\section{EVALUACIÓN}

Al plantear la evaluación de una muerte fetal se recomienda tomar en cuenta las siguientes como posibles causas:

\section{Enfermedades maternas crónicas}

\section{Hipertensión}

El mayor riesgo ocurre con la hipertensión crónica superpuesta a preeclampsia básicamente como etiología de abruptio placentae, insuficiencia úteroplacentaria o hemorragia feto-materna. ${ }^{23}$ El sín- 
drome HELLP también está asociado con una alta tasa de muerte fetal. ${ }^{24,25}$

Como hallazgo incidental, se han asociado niveles elevados de alfa-feto-proteína en suero materno en el segundo trimestre con preeclampsia, retardo del crecimiento intrauterino y muerte fetal. ${ }^{26,27}$ Brazetol y cols. reportaron que cerca del 6 al 7\% de los embarazos asociados con elevaciones de alfafeto-proteína en suero materno durante el segundo trimestre terminaron en muerte fetal. ${ }^{26}$

\section{Diabetes mellitus}

En cuanto a la diabetes mellitus, hay una mayor incidencia de muerte fetal en la de tipo II que en la de tipo $\mathrm{I}^{23}$ se desconoce el mecanismo exacto de muerte fetal. Según lo publicado, esto obedece a la mayor edad y a la obesidad presentes en estas pacientes $^{28}$. Algunas posibles explicaciones son las alteraciones en el metabolismo de los carbohidratos y la insuficiencia útero-placentaria secundaria a enfermedad vascular. ${ }^{23}$

\section{Lupus eritematoso sistémico}

En este la muerte fetal anteparto está relacionada con presencia de hipertensión o con síndrome de anticuerpos antifosfolípidos. ${ }^{23,29,30}$ La presencia de anticoagulante lúpico positivo es el principal predictor de muerte fetal. El posible mecanismo de muerte está relacionado con insuficiencia placentaria. El paso de inmunoglobulinas anti Ro y anti La, está asociado con pobre resultado perinatal, básicamente como causa de bloqueo cardíaco unido a las secuelas del mismo. ${ }^{23}$

\section{Patología renal crónica}

El resultado perinatal está relacionado con el grado de falla renal presente en el momento de la concepción. ${ }^{23}$ La presencia de anemia, hipertensión o desarrollo de preeclampsia empobrecen el pronóstico. ${ }^{23,31}$

\section{Enfermedades tiroideas}

El hipertiroidismo no controlado puede producir tirotoxicosis fetal y ser la causa de muerte fetal. ${ }^{23}$
El hipotiroidismo, aunque menos frecuente que el hipertiroidismo como causa de muerte fetal, está relacionado básicamente con el desarrollo de complicaciones como hipertensión y abruptio placentae. ${ }^{23,32}$

\section{Colestasis del embarazo}

Es una rara complicación del embarazo que se presenta en uno de cada mil a diez mil embarazos, se ha reportado una frecuencia de muerte fetal en mujeres con colestasis de 25 a 30 por 1.000 nacimientos. Se desconoce el mecanismo exacto por el cual se desencadena la muerte fetal. ${ }^{23}$

\section{Trombofilias}

Son anormalidades del sistema de coagulación, pueden ser heredadas o adquiridas y usualmente generan fenómenos trombóticos. ${ }^{33,34}$ Cada año se reconocen nuevas trombofilias y su significancia clínica está determinada por la fuerte asociación con riesgo trombótico y su frecuencia en la población. . 34,35 $^{2}$

Algunas de las trombofilias descritas son:

\section{Deficiencia de antitrombina III}

Fue la primera trombofilia heredada reconocida, pero es la menos común. Se hereda como un patrón autosómico dominante y la prevalencia de estado heterocigoto en la población general se calcula en 1:2.000 a 1:5.000. ${ }^{34}$ Existen dos tipos: I y $l l$ (es la más común). ${ }^{36}$

\section{Deficiencia de proteína C}

Fue la segunda trombofilia descrita; se considera como la más frecuente, ${ }^{37}$ con una prevalencia en la población general del 0,15 al 0,8\%. ${ }^{34}$ Se han descrito también dos tipos: tipo 1 que es la más frecuente y tipo $11 .{ }^{36}$

\section{Deficiencia de proteína $S$}

Es la que sirve como cofactor de la proteína C, su prevalencia en la población general se calcula en menos del $1 \% .^{34}$ 


\section{Factor $\mathrm{V}$ de Leiden}

Es heredada de una forma autosómica dominante y varía según la raza y la ubicación geográfica. ${ }^{34,38}$

\section{Polimorfismo del gen G20210A de protrombina}

Es un cambio en la posición 20210 en el gen de protrombina de guanina por arginina que causa aumento de los niveles de protrombina que promueven la generación de trombina. ${ }^{34,36}$

\section{Mutación del gen de la metilentetrahidrofolato reductasa}

Aún no existen estudios concluyentes respecto de esta trombofilia ${ }^{33}$ (tabla 6).

La metilentetrahidrofolato reductasa actúa en la conversión de la homocisteína a metionina cediendo un grupo metilo. El aumento de la homocisteína causa elevación en la presión sanguínea y es un factor independiente en el desarrollo de arteriosclerosis y enfermedad trombótica. ${ }^{33}$

Los hallazgos anatomopatológicos a nivel placentario en pacientes con trombofilias incluyen la presencia de trombos intravasculares, vasculopatía decidual, necrosis isquémica e infartos vellosos. Como probable mecanismo causal se sugieren las anormalidades de la hemostasia, y de la perfusión placentaria. De las pacientes con pérdidas fetales inexplicadas, hasta un $42 \%$ presenta alteraciones en los factores de la coagulación y un 16\% ha tenido una complicación ginecológica mayor secundaria a estas como retardo de crecimiento intrauterino, pre-eclampsia y abruptio placentae. .3,39 $^{33}$

\begin{tabular}{|c|c|c|}
\hline \multicolumn{3}{|c|}{$\begin{array}{c}\text { Tabla } 6 . \text { Trombofilias } \\
\text { y riesgo de muerte fetal }\end{array}$} \\
\hline & OR & IC95\% \\
\hline Todas las trombofilias & 2,8 & $1,5-5,3$ \\
\hline Factor V de Leyden & 1,5 & $0,7-3,6$ \\
\hline Protrombina & 2,3 & $1,3-4,0$ \\
\hline $\begin{array}{l}\text { Gen de la mutilen- } \\
\text { tetrahidrofolato reductasa }\end{array}$ & 1,5 & $0,8-2,8$ \\
\hline Deficiencia de proteína S & 3,2 & $2,4-4,1$ \\
\hline
\end{tabular}

Many y cols. en un estudio de casos y controles (40 casos y 80 controles) encontraron una prevalencia de trombofilias en pacientes con MFI del $42,5 \%$ en comparación con el $15 \%$ en el grupo control. ${ }^{33}$

Preston y cols. en un estudio europeo de cohortes prospectivo con 1.384 pacientes también encontraron aumento de muerte fetal en pacientes con trombofilia (OR 3,6 intervalo de confianza de 1,4 - 9,4); este aumento es mayor en mujeres con defectos combinados (OR 14,3 intervalo de confianza 2,4 - 86)..$^{40}$

Kupferminc y cols. en un estudio de casos y controles con 220 pacientes (110 casos y 110 controles), encontraron una incidencia del $58 \%$ en mujeres con trombofilias que presentaron MFI comparado con $18 \%$ en mujeres sin este factor de riesgo (OR 3,4 intervalo de confianza de 1,0 - 11,9). ${ }^{41}$

En lo relacionado con la significancia clínica de las trombofilias y el manejo de las pacientes afectadas, existe un considerable confusión. ${ }^{34}$ A quienes tienen una trombofilia reconocida se les puede aconsejar tromboprofilaxis para prevenir resultados adversos en el embarazo. Sin embargo, el manejo óptimo de estas pacientes no está claro todavía, aunque ya hay trabajos que recomiendan el uso de heparina o heparinas de bajo peso molecular. ${ }^{34,38,40,42}$ La biblioteca de Cochrane en su revisión afirma que todavía no hay estudios controlados que determinen el efecto de la profilaxis con heparina en el resultado perinatal de mujeres con trombofilia. ${ }^{43}$ En lo que no existe ninguna duda es que a las pacientes con antecedente de MFI se les deben aconsejar pruebas para descartar trombofilias. ${ }^{44,45,46}$ También se recomienda suplencia de ácido fólico más suplemento de vitaminas $\mathrm{B}_{6} \mathrm{y}_{12}$ en pacientes con hiperhomocisteína. ${ }^{42,47}$

\section{Alteraciones del cordón}

Son responsables de una a dos muertes fetales por cada 1.000 nacimientos. ${ }^{48}$ Los posibles mecanismos por los cuales los accidentes del cordón pueden causar la muerte fetal son: cese del flujo sanguíneo 
(prolapso del cordón), disrupción intermitente del flujo sanguíneo (prolapso parcial, hipoxia, acidosis, falla cardíaca, hipotensión, arritmia, paro cardíaco), pérdida sanguínea fetal y disrupción del flujo (como ocurre en la ruptura velamentosa de la vena umbilical o vasa previa). ${ }^{48,49}$ Otros mecanismos son isquemia uterina y anormalidades en el cordón como arteria umbilical única, cordón corto $(<35 \mathrm{~cm})$, cordón largo $(>80 \mathrm{~cm})$, nudos verdaderos, aneurismas, extremos hiper-helicoidales e hipo-helicoidales, circulares ajustadas y sueltas, y anormalidades en la inserción de la placenta. ${ }^{48}$

\section{Alteraciones citogenéticas}

Los mosaicismos confinados a la placenta se encuentran aproximadamente en el 1 al 2\% de las placentas, lo que significa la presencia de un feto euploide con un mosaicismo aislado en la placenta. ${ }^{49}$ Hay dos mecanismos para que este fenómeno ocurra: uno durante el desarrollo de la mórula y otro segundo error durante la anafase tardía. ${ }^{50}$

\section{Patología infecciosa}

Las infecciones pueden causar muerte fetal por varios mecanismos que incluyen infección directa, daño placentario y enfermedad materna severa. ${ }^{51} \mathrm{La}$ infección bacteriana con o sin membranas íntegras con bacterias como la Escherichia coli, estreptococo del grupo B y Ureaplasma urealyticum son las que más frecuentemente causan muerte fetal. Posteriormente están las del complejo PRACHET antes TORSCH (parvovirus, rubéola, virus de la inmunodeficiencia humana, citomegalovirus, hepatitis, toxoplasmosis y sífilis); también están involucradas la malaria, leptospirosis y las causadas por Listeria monocytogenes. ${ }^{51}$

\section{Tóxicos ambientales}

Durante el proceso de tratamiento de aguas públicas con reactivos de cloro se producen subproductos llamados trialometanos. King y cols. encontraron una fuerte asociación entre la exposición al bromodiclorometano (cantidades mayores a $20 \mathrm{mcg} / \mathrm{l})$ y muerte fetal inexplicada (RR 1,98 IC95\% $1,23-3,49) .{ }^{17}$

\section{Otras causas}

Los desórdenes mendelianos o las patologías bioquímicas son causa de muerte fetal. ${ }^{50}$ Trastornos metabólicos autosómicos recesivos como hemoglobinopatías, enfermedades del glucógeno, alteraciones de aminoácidos y deficiencias de peroxidasas, también se encuentran asociadas como agentes etiológicos de la muerte fetal. ${ }^{49}$

\section{RECOMENDACIONES}

Para ayudar a determinar la etiología en un caso de muerte fetal se han emitido algunas recomendaciones de pasos secuenciales. ${ }^{2}$

1. Realizar una historia clínica lo más completa posible, con especial énfasis en la historia obstétrica, antecedentes familiares y patológicos crónicos, uso de medicamentos y en las pérdidas previas.

2. Evaluación de los controles prenatales realizados y de las valoraciones ecográficas respecto del peso fetal, anomalías anatómicas e índice de líquido amniótico.

3. Al momento del nacimiento se debe realizar una evaluación de la presencia de malformaciones gruesas del niño, la placenta, el cordón umbilical y las membranas.

4. Evaluación histopatológica del feto, cordón, membranas y placenta. Este reporte provee la mayor información a través de la cual es posible detectar la causa de muerte.

5. Evaluación del cariotipo fetal si se encuentran anomalías anatómicas. En caso de maceración marcada se recomienda tomar la muestra de tejido del cordón umbilical o de las membranas.

6. Selección para infecciones congénitas (toxoplasmosis, rubéola, herpes virus, citomegalovirus, sífilis), o algunas otras menos frecuentes (parvovirus), dependiendo del caso individual de cada paciente.

7. Determinación del anticoagulante lúpico y anticuerpos anticardiolipina (casos individuales).

8. Determinación de la prueba de KleihauerBetke, la cual sirve para determinar el volumen 
de transfusión feto-materna en pacientes $\mathrm{Rh}$ negativos (casos individuales).

9. Evaluación de las alteraciones hematológicas (trombofilias en casos individuales) y no descartar posibles alteraciones medio-ambientales.

Las pacientes con antecedentes de MFI deben recibir consejería preconcepcional para corregir los factores de riesgo mencionados y una vez se embaracen se les debe recomendar pruebas de bienestar fetal desde la semana 28 .

Continuidad en los estudios y considerar otras posibilidades apartes de las ya anotadas, es la tarea para seguir en la frontera del conocimiento del problema planteado.

\section{REFERENCIAS}

1. Froen JF, Arnestad M, Frey K, Vege A, Saugstad OD, Stray-Pedersen B. Risk factors for sudden intrauterine unexplained death: epidemiologic characteristics of singleton cases in Oslo, Norway, 1986-1995. Am J Obstet Gynecol 2001;184:694-702.

2. Incerpi MH, Miller DA, Samadi R, Settlage RH, Goodwin TM. Stillbirth evaluation: what tests are needed? Am J Obstet Gynecol 1998;178:1121-5.

3. Cnattingius S, Stephansson O. The epidemiology of stillbirth. Semin Perinatol 2002;26:25-30.

4. Cnattingius S, Forman MR, Berendes HW, Italo L. Delayed childbearing and risk of adverse perinatal outcome. A population-based study. JAMA 1992;268:886-90.

5. Fretts RC, Schmittdiel J, McLean FH, Usher RH, Goldman MB. Increased maternal age and the risk of fetal death. N Engl J Med 1995;333:953-7.

6. Fretts RC, Usher RH. Causes of fetal death in women of advanced maternal age. Obstet Gynecol 1997;89:40-5.

7. Raymond EG, Cnattingius S, Kiely JL. Effects of maternal age, parity and smoking on the risk of stillbirth. Br J Obstet Gynaecol 1994;101:301-6.

8. Sims MA, Collins KA. Fetal death. A 10-year retrospective study. Am J Forensic Med Pathol 2001;22:261-5.

9. Nybo Andersen AM, Wohlfahrt J, Christens P, Olsen J, Melbye M. Maternal age and fetal loss: population based register linkage study. BMJ 2000;320:1708-12.
10. Olausson PO, Cnattingius S, Haglund B. Teenage pregnancies and risk of late fetal death and infant mortality. Br J Obstet Gynaecol 1999;106:116-21.

11. Huang DY, Usher RH, Kramer MS, Yang H, Morin L, Fretts RC. Determinants of unexplained antepartum fetal deaths. Obstet Gynecol 2000;95:215-21.

12. Cnattingius S, Bergstrom R, Lipworth L, Kramer MS. Prepregnancy weight and the risk of adverse pregnancy outcomes. N Engl J Med 1998;338:147-52.

13. Wolfe $\mathrm{H}$. High prepregnancy body-mass index - a maternal-fetal risk factor honor. N Engl J Med 1998;338:191-2.

14. Stephansson O, Dickman PW, Johansson A, Cnattingius S. Maternal weight, pregnancy weight gain, and the risk of antepartum stillbirth. Am J Obstet Gynecol 2001;184:463-9.

15. Fifer WP, Myers MM. Sudden fetal and infant deaths: shared characteristics and distinctive features. Semin Perinatol 2002;26:89-96.

16. Yudkin PL, Wood L, Redman CW. Risk of unexplained stillbirth at different gestational ages. Lancet 1987:1:1192-4.

17. King WD, Dodds L, Allen AC. Relation between stillbirth and specific chlorination by-products in public water supplies. Environ Health Perspect 2000;108:883-6.

18. Cnattingius S, Berendes HW, Forman MR. Do delayed childbearers face increased risks of adverse pregnancy outcomes after the first birth? Obstet Gynecol 1993;81:512-6.

19. Stephansson O, Dickman PW, Johansson A, Cnattingius S. Maternal hemoglobin concentration during pregnancy and risk of stillbirth. JAMA 2000;284:2611-7.

20. Pharoah PO, Ady Y. Consequences of in-utero death in a twin pregnancy. Lancet 2000;355:1597-602.

21. Wisborg K, Kesmodel U, Bech BH, Hedegaard M, Henriksen TB. Maternal consumption of coffee during pregnancy and stillbirth and infant death in first year of life: prospective study. BMJ 2003;326:420.

22. Smith GC, Pell JP, Dobbie R. Caesarean section and risk of unexplained stillbirth in subsequent pregnancy. Lancet 2003:362:1779-84.

23. Simpson LL. Maternal medical disease: risk of antepartum fetal death. Semin Perinatol 2002;26:42-50.

24. Martin JN Jr, Rinehart BK, May WL, Magann EF, Terrone DA, Blake PG. The spectrum of severe 
preeclampsia: comparative analysis by HELLP (hemolysis, elevated liver enzyme levels, and low platelet count) syndrome classification. Am J Obstet Gynecol 1999;180:1373-84.

25.Visser W, Wallenburg HC. Temporising management of severe preeclampsia with and without the HELLP syndrome. Br J Obstet Gynaecol 1995;102:111-7.

26. Brazetol WF, Grover S, Donnenfeld AE. Unexplained elevated maternal serum alpha fetoprotein levels and perinatal outcome in an urban clinic population. Am J Obstet Gynecol 1994;171:1030-5.

27. Krause TG, Christens P, Wohlfahrt J, Lei U, Westergaard T, Nergaard-Pedersen B, et al. Second trimester maternal serum alpha-fetoprotein and risk of adverse pregnancy outcome. Obstet Gynecol 2001;97:277-82.

28. Cundy T, Gamble G, Townend K, Henley PG, MacPherson P, Roberts AB. Perinatal mortality in Type 2 diabetes mellitus. Diabet Med 2000;17:33-9.

29.Lockshin MD. Antiphospholipid antibody. Babies, blood clots, biology. JAMA 1997;277:1549-51.

30. Oshiro BT, Silver RM, Scott JR, Yu H, Brand DW. Antiphospholipid antibodies and fetal death. Obstet Gynecol 1996;87:489-93.

31. Cunningham FG, Cox SM, Harstad TW, Mason RA, Pritchard JA. Chronic renal disease and pregnancy outcome. Am J Obstet Gynecol 1990;163:453-9.

32.Leung AS, Millar LK, Koonings PP, Montoro M, Mestman JH. Perinatal outcome in hypothyroid pregnancies. Obstet Gynecol 1993;81:349-53.

33. Many A, Elad R, Yaron Y, Eldor A, Lessing JB, Kupferminc MJ. Third-trimester unexplained intrauterine fetal death is associated with inherited thrombophilia. Obstet Gynecol 2002;99:684-7.

34. Saade GR, McLintock C. Inherited thrombophilia and stillbirth. Semin Perinatol 2002;26:51-69.

35. Rey E, Kahn SR, David M, Shrier I. Thrombophilic disorders and fetal loss: a meta-analysis. Lancet 2003;361:901-8.

36. Perry SL, Ortel TL. Clinical and laboratory evaluation of thrombophilia. Clin Chest Med 2003;24:153-70.

37. Seligsohn U, Lubetsky A. Genetic susceptibility to venous thrombosis. N Engl J Med 2001;344:1222-31.

38. Martinelli I, Taioli E, Cetin I, Marinoni A, Gerosa S, Villa MV, et al. Mutations in coagulation factors in women with unexplained late fetal loss. N Engl J Med 2000;343:1015-8.

39. Roberts D, Schwartz RS. Clotting and hemorrhage in the placenta a delicate balance. N Engl J Med 2202;347:57-9.

40. Preston FE, Rosendaal FR, Walker ID, Briet E, Berntorp E, Conard J, et al. Increased fetal loss in women with heritable thrombophilia. Lancet 1996;348:913-6.

41. Kupferminc MJ, Eldor A, Steinman N, Many A, BarAm A, Jaffa A, et al. Increased frequency of genetic thrombophilia in women with complications of pregnancy. N Engl J Med 1999;340:9-13.

42. Gebhardt GS, Hall DR. Inherited and acquired thrombophilias and poor pregnancy outcome: should we be treating with heparin? Curr Opin Obstet Gynecol 2003;15:501-6.

43. Walker MC, Ferguson SE, Allen VM. Heparin for pregnant women with acquired or inherited thrombophilias (Cochrane Review). The Cochrane Library, Issue 4, 2004.

44. Sibai BM. Thrombophilias and adverse outcomes of pregnancy — what should a clinician do? N Engl J Med 1999;340:50-2.

45. Greer IA. The challenge of thrombophilia in maternal-fetal medicine. N Engl J Med 2000;342:424-5.

46. Alonso A, Soto I, Urgelles MF, Corte JR, Rodriguez MJ, Pinto CR. Acquired and inherited thrombophilia in women with unexplained fetal losses. Am J Obstet Gynecol 2002;187:1337-42.

47. Jilma B, Kamath S, Lip GYH. ABC of antithrombotic therapy. Antithrombotic therapy in special circumstances. II-In children, thrombophilia, and miscellaneous conditions. BMJ 2003;326:93-6.

48. Collins JH. Umbilical cord accidents: human studies. Semin Perinatol 2002;26:79-82.

49. Lee W, Lee VL, Kirk JS, Sloan CT, Smith RS, Comstock $\mathrm{CH}$. Vasa previa: prenatal diagnosis, natural evolution and clinical outcome. Obstet Gynecol 2000;95:572-6.

50. Wapner RJ, Lewis D. Genetics and metabolic causes of stillbirth. Semin Perinatol 2002;26:70-4.

51. Goldenberg RL, Thompson C. The infectious origins of stillbirth. Am J Obstet Gynecol 2003;189:861-73. 